\title{
SPECTROSCOPIC ELLIPSOMETRIC INVESTIGATION OF THE CLEAN AND OXYGEN EXPOSED Ni(110) SURFACE
}

\author{
L.J. HANEKAMP, S.J.H. BRADER and G.A. BOOTSMA ${ }^{\dagger}$ \\ Department of Apphed Physics, Twente University of Technology, P.O. Box 217, 7500 AE Enschede, \\ The Netherlands
}

Received 5 January 1983; accepted for publication 7 June 1983

\begin{abstract}
Dynamical ellipsometric investigations of the initial oxidation of the $\mathrm{Ni}(110)$ surface have been performed. Ellipsometry appears to be well suited to distinguish between chemisorbed oxygen and nickel oxide on the surface. Annealing at $740 \mathrm{~K}$ causes the nucleation of nickel oxide to proceed faster than at $570 \mathrm{~K}$. After equal exposures however, the nucleation is slower with an oxygen pressure of $2 \times 10^{-7}$ Torr than with a pressure of $1.3 \times 10^{-8}$ Torr. Spectroscopic ellipsometric measurements $(400-800 \mathrm{~nm})$ have been performed on clean and oxygen-exposed (at saturation) $\mathrm{Ni}(110)$ surfaces. The complex dielectric function of the clean surface has been determined. To explain the results of the oxygen exposed surface a model is discussed - a surface layer with a thickness of $6 \dot{\mathrm{A}}$ and a mixture of $90 \% \mathrm{NiO}$ and $10 \% \mathrm{Ni}$ - in which changes with substrate optical properties are taken into account.
\end{abstract}

\section{Introduction}

Ellipsometry is a suitable technique to monitor the interaction between a gas and a solid surface without interference with the processes under investigation (cf. refs. [1,2]).

With a spectroscopic ellipsometer the optical constants of a clean surface can be determined as a function of wavelength, while measurements on adsorbed layers supply data to interpret the surface layer (cf. ref. [2]).

The interaction of oxygen with nickel single crystal surfaces has been investigated by many authors using different surface techniques as LEED, RHEED, AES, EELS, X-ray emission, XPS, UPS, RBS and ellipsometry. Mitchell et al. [3] studied the initial oxidation of the Ni(110) surface by RHEED and X-ray emission. They described this oxidation as a non-activated chemisorption followed by nucleation of an oxide displaying a saturation coverage of two monolayers (one monolayer containing $0.030 \mu \mathrm{g} / \mathrm{cm}^{2}(1.14 \times$ $10^{15}$ atoms $/ \mathrm{cm}^{2}$ ) oxygen). Norton et al. [4] investigated the interaction of

$\dagger$ Deceased. 
oxygen with the $\mathrm{Ni}(110)$ surface by means of photoelectron spectroscopy. They also distinguished two distinct steps: a fast dissociative chemisorption process followed by oxide nucleation and lateral oxide growth to a limiting coverage of three monolayers. However, they take one monolayer to involve $0.80 \times 10^{15}$ oxygen ions $/ \mathrm{cm}^{2}$.

With Rutherford backscattering measurements (RBS), Smeenk et al. [5] determined a saturation coverage of two monolayers, using Mitchell's monolayer definition [3]. The same authors [3-5] also investigated the oxidation of the Ni(110) surface at sample temperatures of around $475 \mathrm{~K}$.

The initial oxidation of nickel has been investigated ellipsometrically only with the Ni(100) surface using a wavelength of $632.8 \mathrm{~nm} \mathrm{[6].} \mathrm{The} \mathrm{plot} \mathrm{of} \mathrm{the}$ relative oxygen Auger signal as a function of the ellipsometric parameter $\delta \Delta$ consists of two linear parts of different slopes. This is in contrast with analogous measurements on copper surfaces [1], where a simple linear relationship was observed.

Spectroscopic ellipsometric measurements on the clean and oxygen exposed $\mathrm{Cu}(110)$ surface showed a dependence of the ellipsometric parameters $\Delta$ and $\psi$ on the azimuth of the plane of incidence [2]. We therefore chose the $\mathrm{Ni}(110)$ surface to perform a spectroscopic ellipsometric investigation of the initial oxidation.

In this paper we report:

(i) Spectroscopic ellipsometric measurements $(400-800 \mathrm{~nm})$ on the clean nickel surface at two azimuths of the plane of incidence.

(ii) Dynamical ellipsometric measurements during oxygen exposure of the nickel surface. The exposures have been performed at sample temperatures of $300,425,475$ and $510 \mathrm{~K}$. Exposure to oxygen was done at $1.3 \times 10^{-8}$ and $2.0 \times 10^{-7}$ Torr. Combined ellipsometric and Auger measurements were carried out after adsorption of increasing amounts of oxygen by the nickel surface at room temperature. These measurements are all performed at $632.8 \mathrm{~nm}$ on crystals which have previously been annealed at 570 or $740 \mathrm{~K}$.

(iii) Spectroscopic ellipsometric measurements on the oxygen exposed nickel surface after reaching a constant value of the ellipsometric parameters during exposure. These investigations are performed at sample temperatures of 300 and $425 \mathrm{~K}$ and two azimuths of the plane of incidence.

\section{Experimental}

The experiments were performed in an ion-pumped stainless steel UHV system with facilities for AES/LEED and ellipsometry. The vacuum system and ellipsometer were essentially the same as described in refs. [2,7].

The single crystal was cut by spark erosion from a $5 \mathrm{~N}$ nickel rod (Metal Crystals Ltd.) to within $0.5^{\circ}$ of the (110) orientation. The specimen was 
mechanically and electrochemically polished and subsequently mounted in the sample holder. The observed sulphur and chlorine contaminations could easily be removed by argon ion bombardment $\left(6 \times 10^{-5} \mathrm{Torr}, 1000 \mathrm{eV}\right)$. The oxygen contamination was removed after several bombardment cycles. The carbon contamination dropped after switching off the ionization gauge in the UHV system during bombardment. The carbon could totally be removed by cycles of heating $\left(740 \mathrm{~K}, \frac{1}{2} \mathrm{~h}\right)$, bombardment during cooling $\left(6 \times 10^{-5}\right.$ Torr, $\left.600 \mathrm{eV}\right)$, oxygen exposure $\left(10^{-6}\right.$ Torr, $\left.\frac{1}{2} \mathrm{~min}\right)$, heating $\left(740 \mathrm{~K}, \frac{1}{2} \mathrm{~h}\right)$ and bombarding $\left(6 \times 10^{-5}\right.$ Torr, $\left.\frac{1}{2} \mathrm{~h}\right)$ during cooling.

As reported by Oda et al. [7] temperature-dependent peaks (TDP) in the secondary electron emission spectrum of copper influence the detection limit of carbon on copper with AES at room temperature. TDP's were also observed with nickel [8] at the same energy values as reported by Becker and Hagstrum [9]. At $740 \mathrm{~K}$ the TDP's were totally absent, except for a small shoulder around $200 \mathrm{eV}$. At $740 \mathrm{~K}$ no contaminations were detected.

\section{Results}

\subsection{Clean nickel surface}

Spectroscopic ellipsometric investigation of the clean surface yields parameters $\bar{\Delta}$ and $\bar{\psi}$. From these the complex optical constant, $\tilde{n}$, and complex dielectric constant, $\tilde{\epsilon}$, given by

$\tilde{n}=n-\mathrm{i} k$,

$\tilde{\boldsymbol{\epsilon}}=e^{\prime}-\mathrm{i} \epsilon^{\prime \prime}=n^{2}-k^{2}-\mathrm{i} 2 n k$,

can be calculated, assuming the reflecting system to be isotropic. For clean $\mathrm{Ni}(110)$ at room temperature $\epsilon^{\prime}$ and $\epsilon^{\prime \prime}$ are given in fig. 1 . The curves in this figure are fits of the values calculated at intervals of $20 \mathrm{~nm}$. The deviations of the individually calculated points are at most 0.08 for $\epsilon^{\prime}$ and 0.05 for $\epsilon^{\prime \prime}$.

The ellipsometric parameters $\bar{\Delta}$ and $\bar{\psi}$ were determined at $\Omega \approx 0^{\circ}$ and $90^{\circ}$, where $\Omega$ is the azimuth of the plane of incidence $\left(\Omega=0^{\circ}:\right.$ plane of incidence parallel to [11 10 ] direction of $\mathrm{Ni}(110)$ surface). In contrast with the results for $\mathrm{Cu}(110)$ [2], these measurements appeared to be independent of the azimuth $\Omega$ in the wavelength region studied within the experimental errors in the absolute values of $\bar{\Delta}$ and $\bar{\psi}$.

The measurements were also performed at a sample temperature of $425 \mathrm{~K}$. Raising the sample temperature brought about maximum changes of 0.12 and 0.18 for $\epsilon^{\prime}$ and $\epsilon^{\prime \prime}$, respectively.

For comparison, literature values of dielectric constants of less-well defined surfaces and samples of nickel at room temperature are also given in fig. 1. The accuracy of these points is limited, as they are mainly obtained from published 

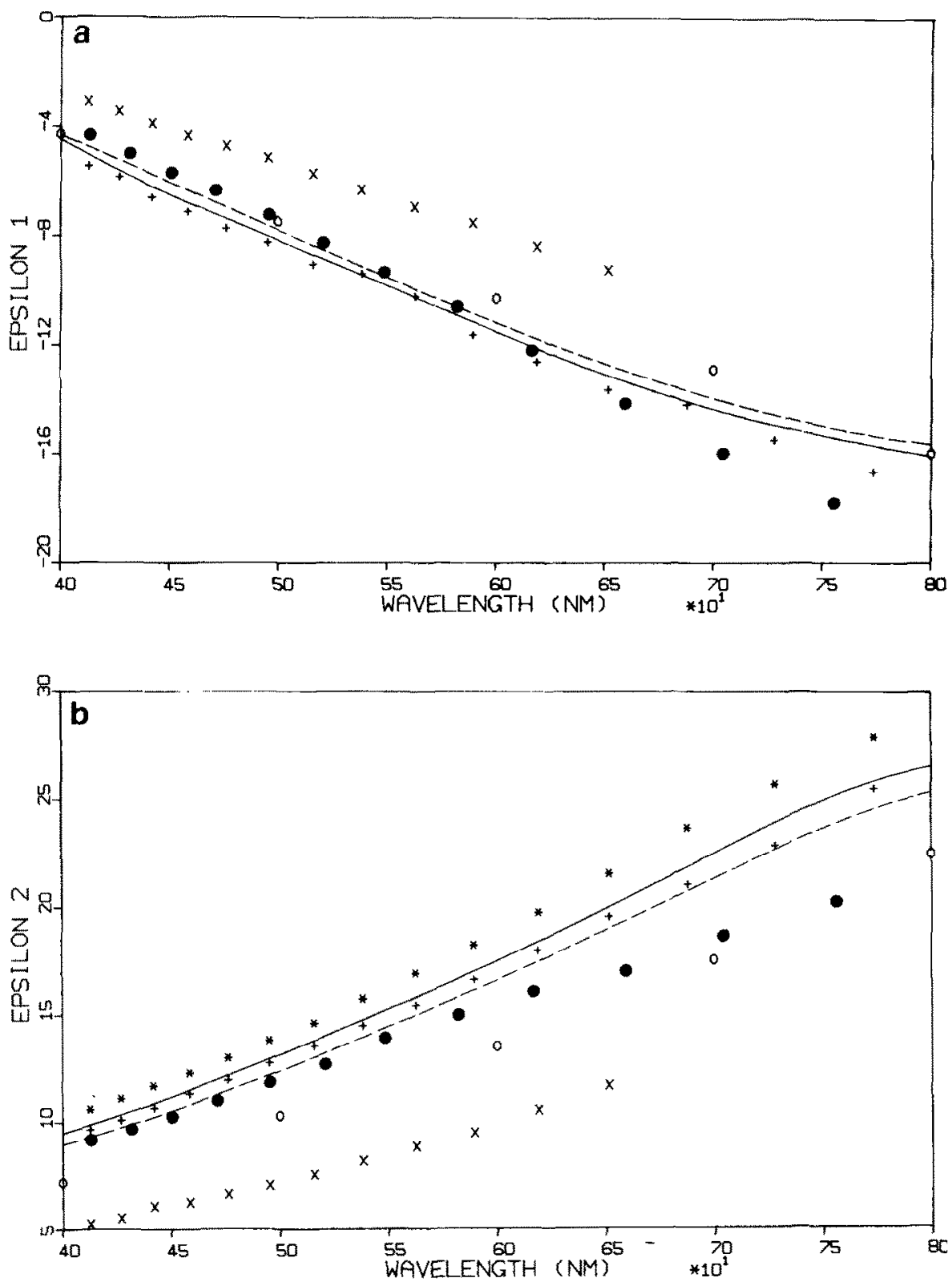

Fig. 1. The components of the complex dielectric function (a) $e^{\prime}$ ("epsilon 1") and (b) $\epsilon^{\prime \prime}$ ("epsilon 2") at room temperature. The calculated values for the clean surface are presented by solid lines, and for the oxygen exposed surface by dashed lines. The values from the literature are given by: (O) ref. [10], (*) ref. [11], (๑) ref. [12], $(x)$ ref. [13] and $(+)$ ref. [14], see text. 
figures. Roberts [10] determined the optical properties of annealed and electropolished polycrystalline nickel samples in vacuum by means of ellipsometry. The nickel was prepared from nickel carbonyl and was vacuum melted. Shiga and Pells [11] obtained $\epsilon^{\prime \prime}$ by means of adsorption spectra measurements of a polycrystalline sample thermally cleaned at $770 \mathrm{~K}$ in ultrahigh vacuum. This sample was prepared from $99.999 \%$ deoxidized nickel powder by argon arc melting. After annealing at $1300 \mathrm{~K}$ in vacuum the sample was electropolished. The results of Johnson and Christy [12] were collected from reflection and transmission measurements on vacuum-evaporated, annealed films, which had been exposed to air. Studna [13] reports the results of measurements on a $\mathrm{Ni}(110)$ surface with a high-resolution spectroscopic ellipsometer. The purity of the nickel was $99.995 \%$. The surface was prepared by a mechanical and an optical polish. The measurements were performed at a base pressure of $5 \times 10^{-7}$ Torr. With spectroscopic ellipsometry Stoll and Jung [14] determined $\epsilon^{\prime}$ and $\epsilon^{\prime \prime}$ of a high-quality Ni(110) single crystal. The sample was polished by hand and subjected to a thermal treatment $(700 \mathrm{~K})$ under UHV conditions.

\subsection{Interaction of oxygen with the clean nickel surface}

In fig. 2 the change in the ellipsometric parameter $\Delta(\delta \Delta=\bar{\Delta}-\Delta$ with $\bar{\Delta}$ : clean surface) upon oxygen exposure is given. The curves are measured at

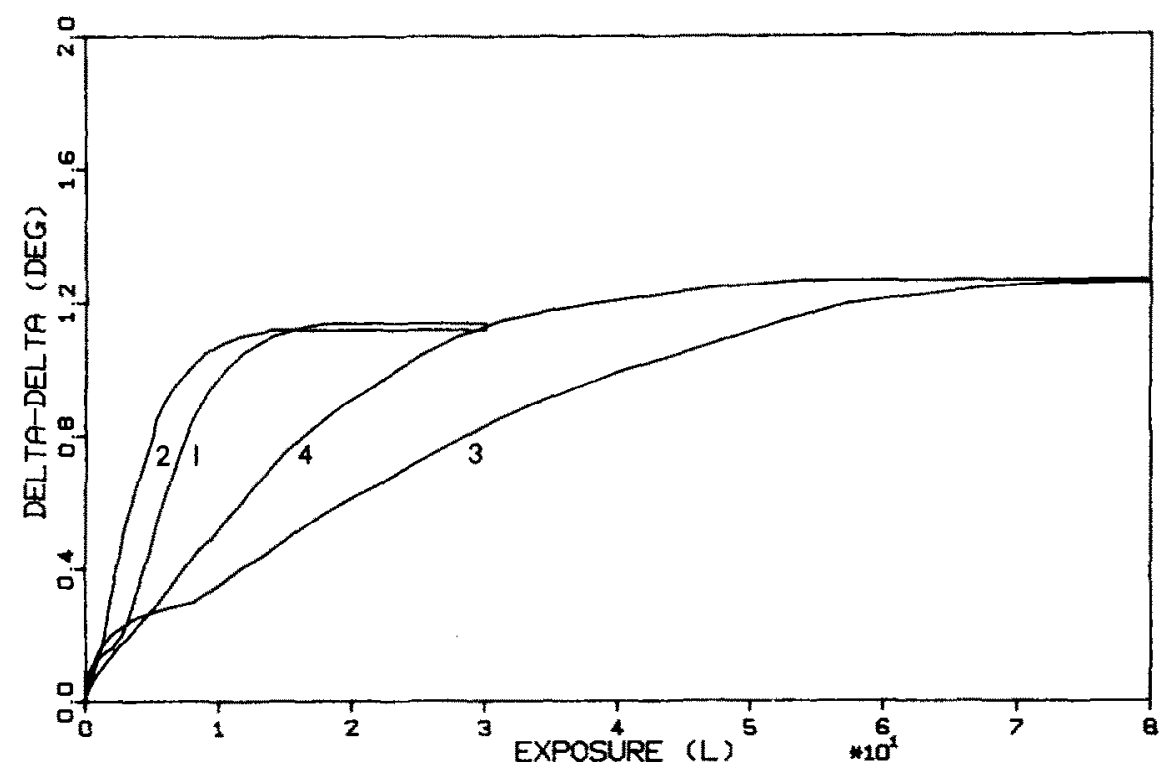

Fig. 2. $\delta \Delta$ ("delta-delta") upon oxygen exposure at $1.3 \times 10^{-8}$ Torr. Curves 1,2 and 3, 4 are taken at sample temperatures of 300 and $425 \mathrm{~K}$ respectively. Annealing temperatures are 570 and $740 \mathrm{~K}$ for the curves 1,3 and 2, 4, respectively. Wavelength $632.8 \mathrm{~nm} ; \phi=66^{\circ}$. 


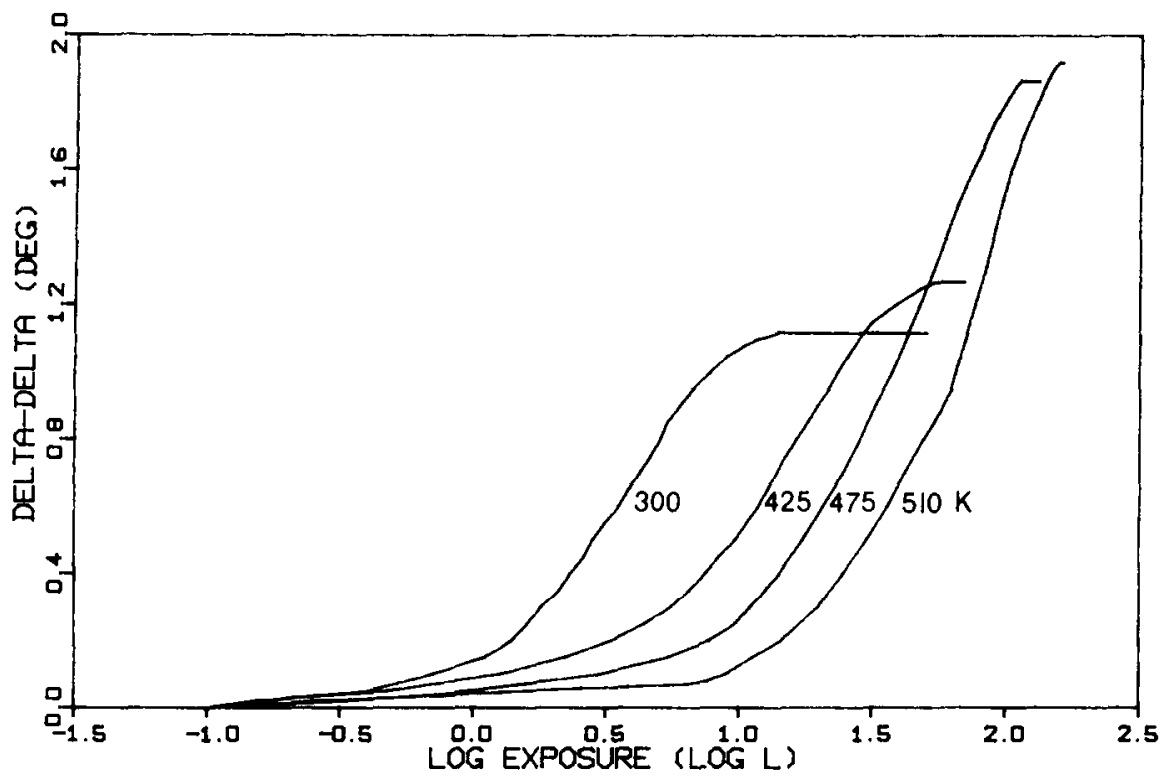

Fig. 3. $\delta \Delta$ ("delta-delta") upon oxygen exposure at $1.3 \times 10^{-8}$ Torr as a function of sample temperature. Annealing temperature $570 \mathrm{~K}$.

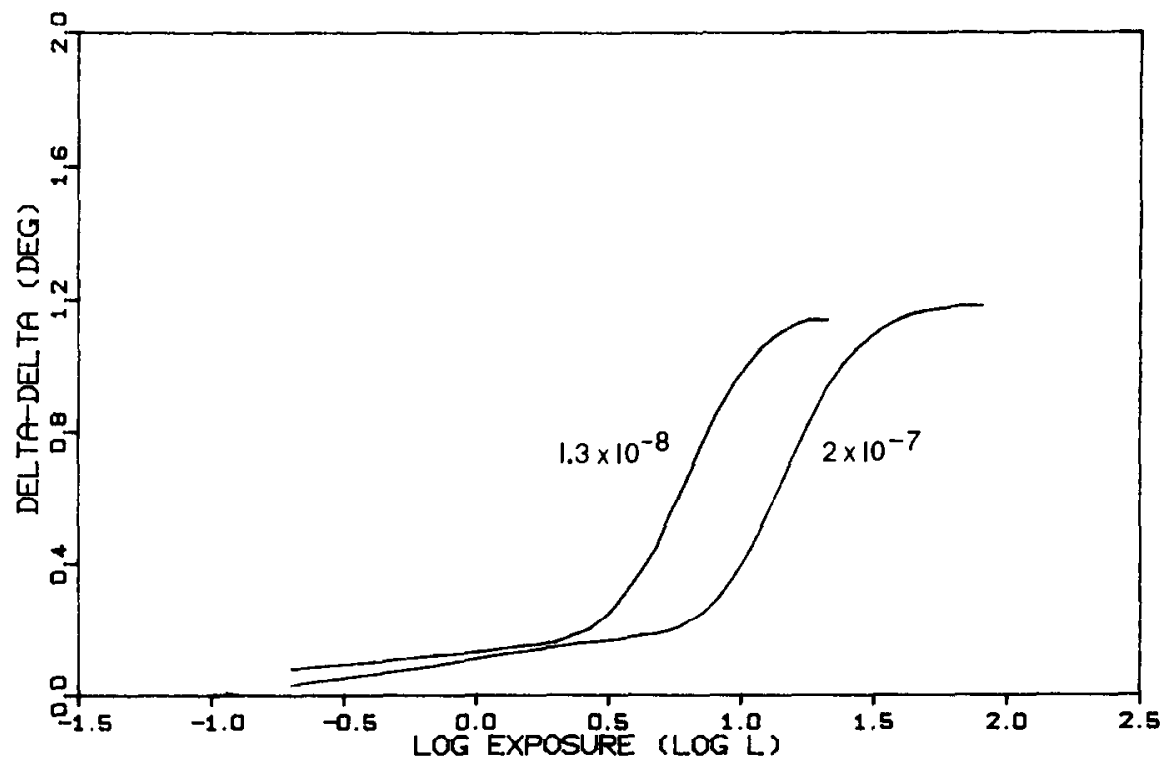

Fig. 4. $\delta \Delta$ ("delta-delta") upon oxygen exposure at $1.3 \times 10^{-8}$ and $2.0 \times 10^{-7}$ Torr. Annealing temperature $570 \mathrm{~K}$. 
different sample temperatures and after a previous annealing treatment at different temperatures. The change in the second ellipsometric parameter, $\delta \psi$ was also measured. During the exposure $\delta \psi$ increased continuously up to a saturation value of about $0.08^{\circ}$. This value was reached at the same time at which the value of $\delta \Delta$ saturated.

To investigate the influence of sample temperature and exposure pressure on the interaction, identical measurements were performed as a function of these two parameters. In fig. $3, \delta \Delta$ upon oxygen adsorption is plotted for different sample temperatures at an oxygen pressure of $1.3 \times 10^{-8}$ Torr. The results at different exposure pressures measured at room temperature are given in fig. 4. With all these measurements the samples had been previously annealed at $570 \mathrm{~K}$.

Fig. 5 shows the normalized oxygen Auger peak heights $\left(h_{\mathrm{O}} / h_{\mathrm{Ni}}\right)$, taken at $515 \mathrm{eV}$ for oxygen and $853 \mathrm{eV}$ for nickel, versus $\delta \Delta$ measured after adsorption of increasing amounts of oxygen by the crystal. The measurements were performed at room temperature on samples which were annealed at 570 and $740 \mathrm{~K}$ respectively. The indicated points were measured in several cycles with exposures in steps.

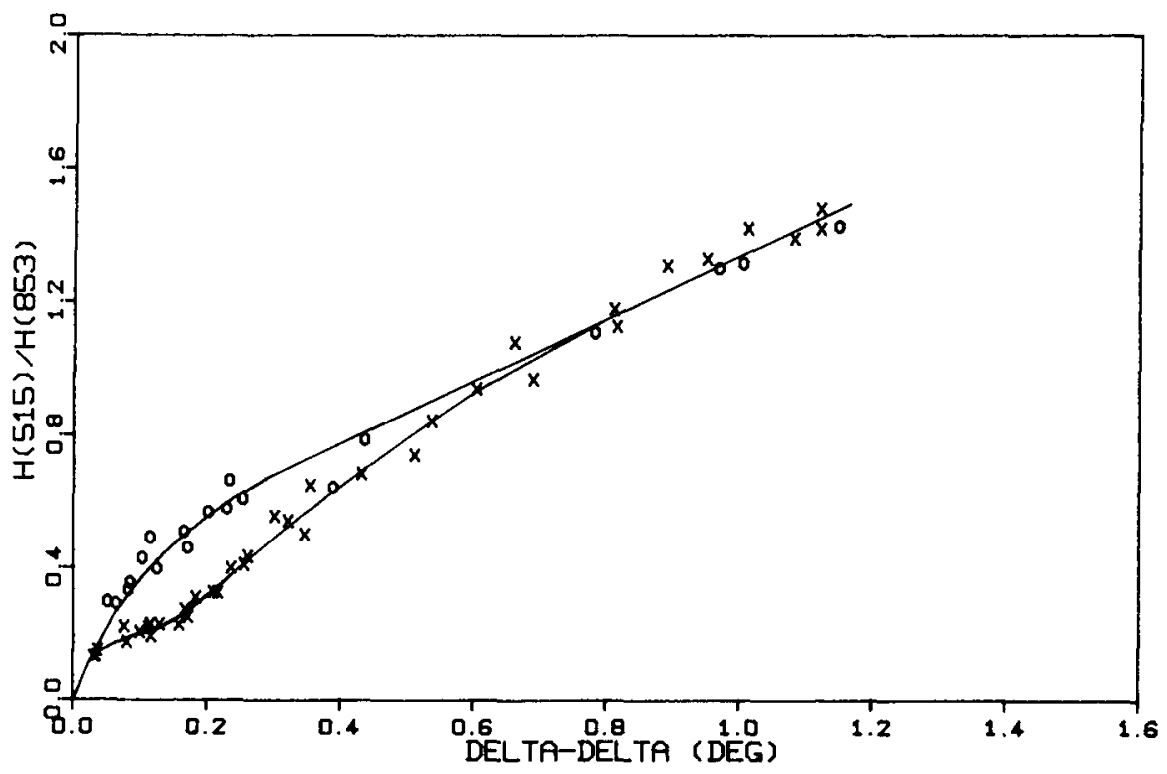

Fig. 5. The normalized oxygen Auger signal $h_{\mathrm{O}}(515 \mathrm{eV}) / h_{\mathrm{Ni}}(853 \mathrm{eV})$ versus $\delta \Delta$ ("delta-delta"), measured at room temperature. Wavelength $632.8 \mathrm{~nm} ; \phi=66^{\circ}$. Annealing temperature: $(X) 570$ $\mathrm{K}$, (O) $740 \mathrm{~K}$. 


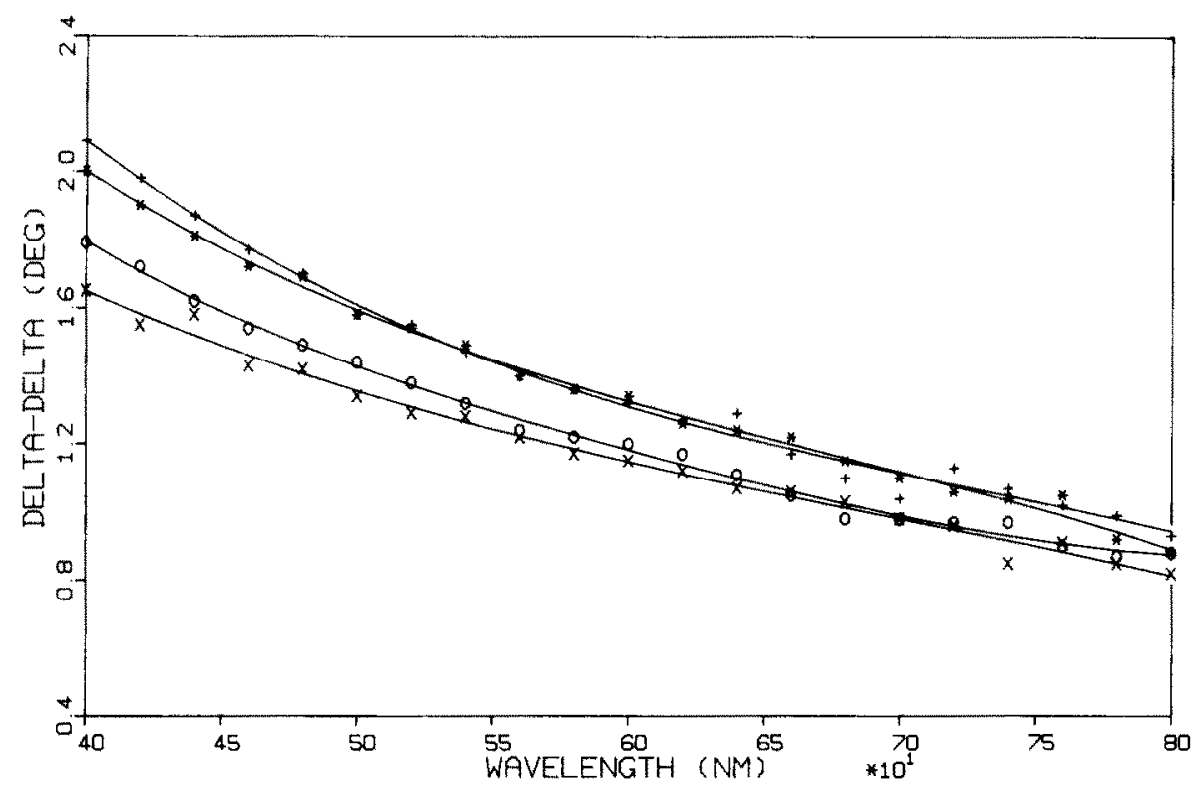

Fig. 6. Ellipsometric effect ("delta-delta") as a function of wavelength upon oxygen adsorption on nickel in the saturation stage: $(\times),(O)$ at $300 \mathrm{~K}$ for $\Omega \approx 0^{\circ}$ and $90^{\circ}$ respectively; $(*),(+)$ at 425 $\mathrm{K}$ for $\Omega=0^{\circ}$ and $90^{\circ}, \phi=66^{\circ}$.

\subsection{Oxygen covered nickel surface}

The ellipsometric parameters $\delta \Delta$ and $\delta \psi$ reached a saturation value during the initial oxidation of the nickel surface. The saturation stage was obtained for sample temperatures of 300 and $425 \mathrm{~K}$ after 20 and $80 \mathrm{~L}$ oxygen exposure, respectively (cf. fig. 2). The spectroscopic dependence of $\delta \Delta$ and $\delta \psi$ in this stage was measured. In fig. $6, \delta \Delta$ is given for two azimuths of the plane of incidence, $\Omega \approx 0^{\circ}$ and $90^{\circ}$, and for two sample temperatures. The sample temperature during exposure and measurements was the same. The drawn lines are polynominal fits of the measured data, which are also shown.

Also at lower exposures, around $3 \mathrm{~L}$, spectroscopic measurements were carried out at room temperature. It appeared difficult to reproduce these exposures. The shape of the $\delta \Delta$ versus $\lambda$ curves for these states was analogous to that of the saturation stage at room temperature within the experimental error. 


\section{Discussion}

\subsection{Clean nickel surface}

The measured dielectric function data in fig. 1 are in good agreement with the results of Stoll and Jung [14]. The data of Johnson and Christy [12] are different mainly at higher wavelengths. Robert's results [10] show the differences especially for $\epsilon^{\prime \prime}$. Shiga and Pells [11] give only $\epsilon^{\prime \prime}$ values, which are a little larger than our results. The largest discrepancies are with Studna's results [13].

An important reason for the differences between our data and those from the literature may be the way of surface preparation. In section 3.1, a summary is given of the methods used by the different authors. Most of them applied mechanical and electrochemical polishing, also thermal treatment under vacuum conditions was used. But none of the cited authors cleaned the surface by argon ion bombardment and controlled the surface by AES.

As described in section 2, before bombardment the surface was contaminated by oxygen, sulphur, chlorine and carbon. To get an impression concerning the effect of contaminations on the dielectric constants, we have calculated effective values of $\epsilon^{\prime}$ and $\epsilon^{\prime \prime}$ by neglecting the presence of a surface layer. The calculations have been performed with the $\Delta$ and $\psi$ values measured after reaching the saturation stage during oxygen exposure at room temperature (fig. 2). The results, also given in figs. $1 \mathrm{a}$ and $1 \mathrm{~b}$ by a dashed line, show that the observed differences could be explained for a considerable part by the presence of surface contaminations. Another reason for the differences is the variety in physical nature of the sample: single crystals, polycrystalline bulk and films.

Within the experimental error, the dielectric function data appeared to be independent of the azimuth of the plane of incidence in the investigated wavelength region. This is in contrast with the observed optical anisotropy of the $\mathrm{Cu}(110)$ surface [2].

\subsection{Interaction of oxygen with the clean nickel surface}

The initial oxidation of the $\mathrm{Ni}(110)$ surface has been investigated by several authors by measuring the oxygen coverage as a function of exposure. It appears that this oxidation process takes place in two stages (cf. introduction). In our ellipsometric measurements, $\delta \Delta$ and $\delta \psi$ are the recorded parameters. Because the effect in $\delta \psi$ is small this parameter is not further discussed. The ratio between the $\delta \Delta$ values of stages one and two is $\sim 0.14$ at room temperature (fig. 4). This number is not the ratio between the oxygen coverages of the two stages, as $\delta \Delta$ is dependent on the refractive index $n$ and the thickness $d$ of the surface layer. Mitchell et al. [3] and Smeenk et al. [5] calculated for the 
ratio between the monolayers $\sim 0.33$, and Norton et al. $[4] \sim 0.20$, but the last reported a limited saturation coverage of three monolayers instead of two. Bootsma and Meyer [15] proved that even in the submonolayer region the relations between $\delta \Delta, \delta \psi$ and $n, d$ of this layer are correct by introducing an effective layer thickness $\left(d_{\mathrm{m}} \theta\right)$. Here $d_{\mathrm{m}}$ is the thickness of a complete monolayer and $\theta$ the fraction of the occupied positions. Comparison of our results with those from the literature leads to the conclusion that the different stages have different refractive indices (or polarizabilities). The formation of a monolayer of nickel oxide gives a larger ellipsometric effect than a chemisorbed oxygen layer.

From figs. 2 and 4 it can be concluded that the limiting coverage at saluration is independent of the applied annealing temperalures and oxygen exposure pressures. With increasing sample temperatures, $\delta \Delta$ reaches larger values, cf. fig. 3, which can be explained by a further growth in depth. In the first stage $\delta \Delta$ appears, within the experimental error, independent of the applied exposure temperature and proportional to the oxygen exposure pressures, cf. figs. 3 and 4. These observations are in agreement with Mitchell's conclusions [3]. The slopes of the $\delta \Delta$ versus the logarithm of the oxygen exposure curves during stage 2 are almost independent of the applied exposition temperatures and pressures. However this stage starts at higher exposure values at increasing exposition temperatures (fig. 3). An increase of the oxygen exposition pressure causes the same effect (fig. 4). This indicates a delay of the nucleation of the oxide at higher exposition temperatures and pressures.

For the two applied annealing temperatures (570 and $740 \mathrm{~K}$ ) the opposite effect is measured; at the higher annealing temperature more nickel oxide is produced at the same exposure (fig. 2). The path of the curves in this figure suggests that at the lower annealing temperature the nucleation of nickel oxide not only remains behind, but also the starting point of this nucleation is postponed till higher exposures. From fig. 5 it can be concluded that the ratio nickel oxide/chemisorbed oxygen is smaller at the higher annealing temperature for a certain amount of oxygen on the surface. The relative amount of adsorbed oxygen after a previous annealing treatment at a tempcrature of 570 or $740 \mathrm{~K}$ can be derived as a function of the oxygen exposure from the combined results in figs. 2 and 5. More oxygen is adsorbed at higher annealing temperature for a fixed exposure value in the second stage of oxidation.

\subsection{Oxygen covered nickel}

The ellipsometric effect in $\Delta$, caused by oxygen adsorption, as shown in fig. 6 , shows a slight dependence on the azimuth of the plane of incidence at lower wavelengths. In anisotropy investigations with clean surfaces the experimental errors in the window corrections are important because $\delta \bar{\Delta}=\bar{\Delta}\left(\Omega=0^{\circ}\right)-\bar{\Delta}(\Omega$ $=90^{\circ}$ ). Identical measurements on oxygen exposed surfaces are in first order 
independent of window corrections, because $\delta(\delta \Delta)=\delta \Delta\left(\Omega=0^{\circ}\right)-\delta \Delta(\Omega=$ $\left.90^{\circ}\right)$ with $\delta \Delta\left(\Omega=0^{\circ}, 90^{\circ}\right)=\bar{\Delta}\left(\Omega=0^{\circ}, 90^{\circ}\right)-\Delta\left(\Omega=0^{\circ}, 90^{\circ}\right)$. The observed differences in $\delta \Delta$ for the two orientations at shorter wavelengths are therefore significant also because they are obtained at both 300 and $425 \mathrm{~K}$. With the effective medium approximation (EMA) due to Bruggeman [16], a model of the surface layer after reaching a saturation coverage during oxygen exposure at room temperature can be developed, as has been done for $\operatorname{Mg}(001)$ [17] and copper [2].

A detailed description of the EMA has been given by Aspnes et al. [18-20]. The EMA formulation for a medium of two components, where the effective medium itself acts as host medium, is

$f \frac{\epsilon_{1}-\langle\epsilon\rangle}{\epsilon_{1}+2\langle\epsilon\rangle}+(1-f) \frac{\epsilon_{2}-\langle\epsilon\rangle}{\epsilon_{2}+2\langle\epsilon\rangle}=0$

where $\langle\epsilon\rangle, \epsilon_{1}$ and $\epsilon_{2}$ are the complex dielectric functions of the effective medium, components 1 and 2 , and $f$ is the volume fraction of component 1 .

With the computer program of McCrackin [21] the ellipsometric effects caused by an effective layer of $\mathrm{Ni}$ and $\mathrm{NiO}$ mixtures on clean $\mathrm{Ni}$, were calculated. The results are given in fig. 7. The thickness of this layer was chosen equal to $6 \AA$, another choice does not influence the shape but only the

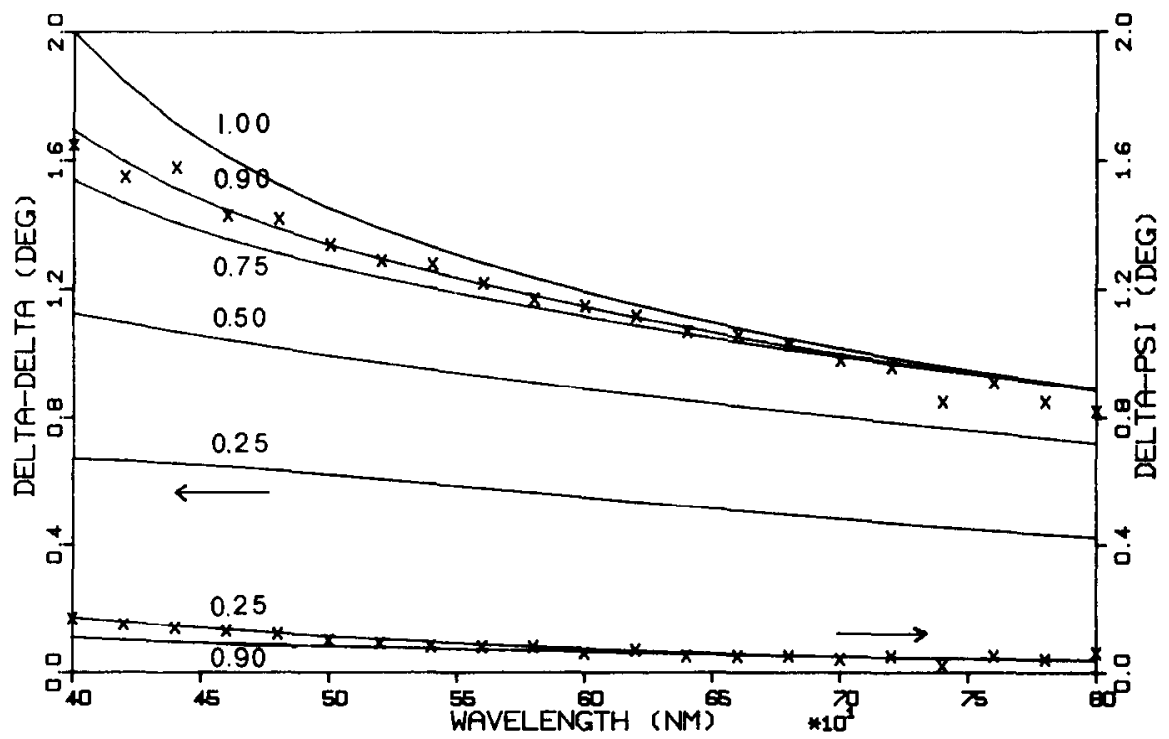

Fig. 7. Ellipsometric effects $\delta \Delta$ ("delta-delta") and $\delta \psi$ ("delta-psi"), calculated for layers of $\mathrm{NiO}$ and $\mathrm{Ni}$ at room temperature on clean $\mathrm{Ni}(110)$. The numbers in the figure indicate the volume fraction of NiO. ( $\times$ ) Measured values after $20 \mathrm{~L}$ oxygen exposure at room temperature. $\phi=66^{\circ}$. 
height of the curves. The effective dielectric functions of the mixtures were calculated with eq. (3). The substituted values of $\epsilon_{1}$ and $\epsilon_{2}$ were derived from the optical constants of $\mathrm{NiO}$ single crystals, published by Powell and Spicer [22], and our measurements on clean nickel.

The results in $\delta \Delta$ (cf. fig. 7) show a good agreement for an effective layer with a thickness of about $6 \AA$ and a composition of $90 \% \mathrm{NiO}$ and $10 \% \mathrm{Ni}$. The influence of the composition of the layer on $\delta \psi$ is relatively small and almost in the order of the experimental errors.

Mitchell et al. [3] report that at the onset of stage two, RHEED experiments indicate the appearance of $\mathrm{NiO}$. The $\mathrm{NiO}$ grows with the epitaxial relationship $\mathrm{NiO}(001)\|\mathrm{Ni}(110), \quad \mathrm{NiO}[1 \overline{1} 0]\| \mathrm{Ni}[1 \overline{1} 0]$.

Nickel oxide crystallizes in the $\mathrm{NaCl}$ structure with $a_{0}=4.17 \AA$. Taking into account the magnitude of the ionic radii we suppose the about $6 \AA$ thick effective layer is consisting of three ionic $\{100\}$ layers NiO. With a composition of $90 \% \mathrm{NiO}$ and $10 \% \mathrm{Ni}$ in the surface layer the oxygen coverage on the surface is $0.08 \mu \mathrm{g} / \mathrm{cm}^{2}$. This is approximately $25 \%$ more than the values reported elsewhere [3-5]. The influence of the presence of an interface of displaced nickel atoms between oxide and substrate, as reported by Smeenk et al., has not been taken into account in our calculations of $\delta \Delta$ and $\delta \psi$.

If we assume no temperature dependence of the dielectric constants of $\mathrm{Ni}$ and $\mathrm{NiO}$ in the temperature interval $300-425 \mathrm{~K}$, the differences in the $\delta \Delta$ curves at 300 and $425 \mathrm{~K}$ can be explained by a further in-depth growth of the $\mathrm{NiO}$ layer. The ratios of the heights of the curves given in fig. 6 , at the two temperatures, are $1.24 \pm 0.02$ and $1.19 \pm 0.04$ for $\Omega=0^{\circ}$ and $90^{\circ}$, respectively. This is an indication of an increase by slightly more than half a monolayer.

\section{Conclusions}

(i) The dielectric function data of Auger clean Ni(110) obtained with spectroscopic ellipsometry show good agreement with those of less defined nickel surfaces published by other authors.

(ii) Dynamical ellipsometric measurements during oxygen exposure on the nickel surface complement other applied surface techniques. The relative change in the ellipsometric parameter $\Delta$ gives information concerning the ratio nickel oxide/chemisorbed oxygen during the nickel oxide nucleation. Exposure pressure and annealing temperature appear to influence the nucleation of nickel oxide. The saturation at the end of this process is, however, independent of these parameters.

(iii) At saturation coverage calculations were performed with the EMA. The best fit for $\delta \Delta$ with the experimental results is obtained with three monolayers of oxygen. The surface layer, with a thickness of $6 \AA$, contains a mixture of $90 \% \mathrm{NiO}$ and $10 \% \mathrm{Ni}$. 


\section{Acknowledgements}

The authors would like to thank Professor J.W. Geus and Dr. A. van Silfhout for helpful discussions. They are also indebted to Mr. Th.L. Schroote (State University of Utrecht) for the preparation of the nickel samples and to Mr. K.O. van der Werf for technical assistance.

\section{References}

[1] F.H.P.M. Habraken and G.A. Bootsma, Surface Sci. 87 (1979) 333.

[2] L.J. Hanekamp, W. Lisowski and G.A. Bootsma, Surface Sci. 118 (1982) 1.

[3] D.F. Mitchell, P.B. Sewell and M. Cohen, Surface Sci. 69 (1977) 310.

[4] P.R. Norton, R.L. Tapping and J.W. Goodale, Surface Sci. 65 (1977) 13.

[5] R.G. Smeenk, R.M. Tromp, N.F. van der Veen and F.W. Saris, Surface Sci. 95 (1980) 156.

[6] P.K. de Bokx, F. Labohm, O.L.J. Gijzeman, G.A. Bootsma and J.W. Geus, Appl. Surface Sci. 5 (1980) 321.

[7] O. Oda, L.J. Hanekamp and G.A. Bootsma, Appl. Surface Sci, 7 (1981) 206.

[8] L.J. Hanekamp, S.J.H. Brader and G.A. Bootsna, to be published.

[9] G.E. Becker and H.D. Hagstrum, J. Vacuum Sci. Technol. 11 (1974) 284.

[10] S. Roberts, Phys. Rev. 114 (1959) 104.

[11] M. Shiga and G.P. Pells, J. Phys. C2 (1969) 1847.

[12] P.B. Johnson and R.W. Christy, Phys. Rev. B9 (1974) 5056.

[13] A.A. Studna, Solid State Commun. 16 (1975) 1063.

[14] M.Ph. Stoll and C. Jung, J. Phys. F (Metal Phys.) 9 (1979) 2491.

[15] G.A. Bootsma and F. Meyer, Surface Sci. 14 (1969) 52.

[16] D.A.G. Bruggeman, Ann. Physik (Leipzig) 24 (1935) 636.

[17] R. Kötz, B. Hayden, E. Schweizer and A.M. Bradshaw, Surface Sci. 112 (1981) 229.

[18] D.E. Aspnes, J.B. Theeten and F. Hottier, Phys. Rev. B20 (1979) 3292.

[19] D.E. Aspnes and J.B. Theeten, Phys. Rev. Letters 43 (1979) 1046.

[20] D.E. Aspnes, E. Kinsbron and D.D. Bacon, Phys. Rev, B21 (1980) 3290.

[21] F.L. McCrackin, FORTRAN Program for Analysis of Ellipsometer Measurements, Natl. Bur. Std. (US) Tech. Note 479 (Natl. Bur. Std., Washington, DC, 1969).

[22] R.J. Powell and W.E. Spicer, Phys. Rev. B2 (1970) 2182. 